\title{
FBMC-based air interface for 5G Mobile: Challenges and proposed solutions
}

\author{
Invited paper \\ Malte Schellmann ${ }^{1}$, Zhao Zhao ${ }^{1}$, Hao Lin ${ }^{2}$, Pierre Siohan ${ }^{2}$, \\ Nandana Rajatheva ${ }^{3}$, Volker Luecken ${ }^{4}$, Aamir Ishaque ${ }^{4}$ \\ ${ }^{1}$ Huawei European Research Center, Munich, Germany \\ ${ }^{2}$ Orange Labs, Rennes, France \\ ${ }^{3}$ Centre for Wireless Communications, University of Oulu, Finland \\ ${ }^{4}$ Institute for Integrated Signal Processing Systems, RWTH Aachen University, Germany
}

\begin{abstract}
Scenarios envisaged for mobile communication systems beyond 2020 imply that future applications and services will impose highly diverse requirements on the system design. To appropriately respond to these requirements while ensuring an efficient usage of available spectrum and system resources, the air interface of $5 \mathrm{G}$ system is expected to provide much more flexibility compared to today's systems. Filterbank based multicarrier (FBMC) may be considered a potential enabler for that purpose, as it provides excellent waveform properties with additional degrees of freedom for the system design, thanks to the use of prototype filters that may be adjusted to meet desired system constraints. This paper elaborates on the challenges that still need to be solved to render FBMC an eligible candidate as fundamental technology for 5G mobile radio and presents some first approaches towards practical solutions.
\end{abstract}

\section{INTRODUCTION}

In today's mobile communication systems, cyclic-prefix-based orthogonal frequency division multiplexing (CP-OFDM) has been widely adopted. The concept of multi-carrier modulation (MCM) is recognized to be an efficient scheme for wideband transmission. Nevertheless, for moving towards future radio systems beyond 2020, some rethinking on the suitability of legacy waveforms is necessary. More specific, the question arises whether the traditional fashion of MCM can effectively meet the demands resulting from the emerging scenarios of future communication. One of the main drawbacks spotted for CP-OFDM is that it cannot provide reasonable degrees of freedom towards flexibility, which would allow to providing, instead of a fixed waveform configured as a best compromise, a more adaptive and thus efficient solution.

On the other hand, filter-bank-based schemes generalize the MCM concept. The key idea is that the modulated signal on each subcarrier is shaped by a well-designed prototype filter, which is different from the traditional rectangular pulse in $\mathrm{CP}$ OFDM with its Sinc-shaped spectrum. This is an important improvement, because it indeed provides a new degree of freedom, which can be used for optimizing the waveform towards various transmission characteristics. In addition, FBMC transmission does not necessarily need a cyclic prefix and therefore achieves full spectral efficiency. More importantly, FBMC is an enabler for efficient spectrum usage thanks to the confined power localization in frequency of the filter. Furthermore, as an outcome from filter bank theory, the overall framework of FBMC modulation/demodulation can be efficiently realized with fast Fourier transforms (FFT) and polyphase filtering [1]. Therefore, FBMC is indeed a favorable alternative to CP-OFDM, providing additional flexibility, robustness, and efficiency. However, to pave the way for FMBC reaching maturity for practical application in the future mobile radio system, some challenges are still remaining. These challenges have been identified by the authors in their collaborative work performed in the EU FP7 METIS project [18]. In this paper, the first approaches and solutions to address these challenges are presented.

This paper is organized as follows: A brief overview on FBMC and related state of the art is given in section II. In section III, scenarios for $5 \mathrm{G}$ mobile radio developed in METIS are used to illustrate that FBMC can be considered a potential enabler to efficiently address the challenges of future communication. In section IV, some open challenges are briefly described and solution proposals are presented. Conclusions are drawn in section $\mathrm{V}$.

\section{FBMC/OQAM: A BRIEF OVERVIEW}

In this section, we give a brief overview on the state of the art of FBMC with Offset-QAM (OQAM) signaling in several respects, such as prototype filter and transceiver algorithm design, as well as related research projects.

\section{A. Filter bank theory}

For a multi-carrier system, denote $F_{0}$ as the spacing between sub-carriers and $T_{0}$ as the symbol duration. The modulated signal can be written as a linear combination of a Gabor family, i.e. the triplet $\left(f, T_{0}, F_{0}\right)$, where $f$ is the waveform pulse or prototype function. Based on the Balian-Low theorem [2], we can deduce that it is not possible to get a modulation scheme holding at the same time the property of (i) orthogonality in the complex field, (ii) a well-localized pulse shape both in time and frequency, and (iii) reaching maximum spectral efficiency by 


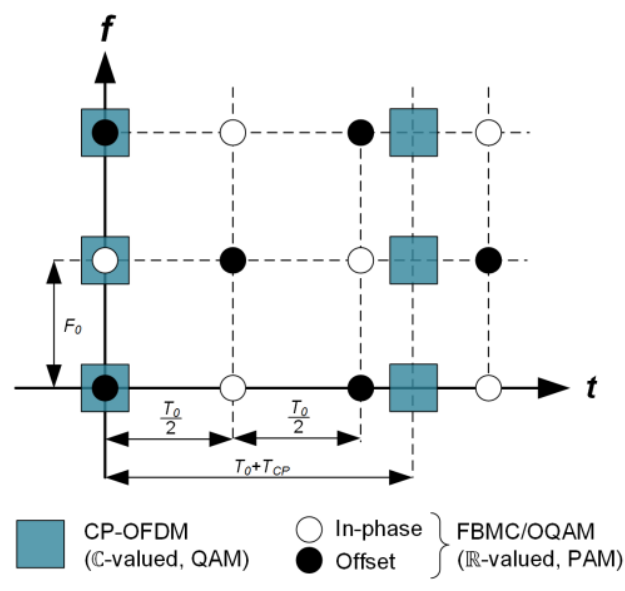

Fig. 1: Time / frequency lattice of CP-OFDM and FBMC/OQAM

keeping Nyquist rate transmission. When CP-OFDM and FBMC/OQAM are compared in the time-frequency lattice representation, as shown in Fig. 1, the CP-OFDM waveform meets the complex orthogonality property, but at the same time only has a poor localization in the frequency domain due to the Sinc-shaped spectrum of the pulse. Moreover, it does not achieve maximum spectral efficiency due to the additional overhead of the CP. On the contrary, FBMC/OQAM schemes relax the complex orthogonality to real field only. Due to their pulse shaping design they can be better localized in time and frequency, eventually depending on the design of $f$. With this flexibility, FBMC/OQAM is able to achieve the maximum spectral efficiency.

\section{B. FBMC prototype filter design}

Currently, there are two major design criteria for the prototype filters in FBMC/OQAM systems, which are either the optimization of time-frequency-localization (TFL) or only frequency localization (FL). The TFL criterion aims to design a waveform that is well-localized both in time and frequency domain, such as in [1], [3]. When the radio coexistence and out-of-band leakage becomes an important metric, as foreseen for future mobile systems, FL criterion needs to be taken into consideration. Several algorithms have been presented for this criterion, e.g. the PHYDYAS filter [4]; the Rossi filter [5] and the frequency selectivity algorithm [6].

\section{FBMC/OQAM transceiver design}

For the FBMC/OQAM transceiver design, many contributions have been achieved in the past years. Due to space limitations, we briefly go through a part of the prior art in the following.

1) Modem implementation: The efficient realization of subband filter chains for FBMC/OQAM is feasible by the use of polyphase networks [7]. With the number of subcarriers, denoted $\mathrm{N}$, the complexity of the FFT is growing with $\mathrm{O}(\mathrm{N} \log \mathrm{N})$. At the same time, the required subband filter grows with $\mathrm{O}(\mathrm{KN})$. Hence, with larger subchannel counts, the basic modulator complexity is only slightly increased in comparison to CP-OFDM schemes.
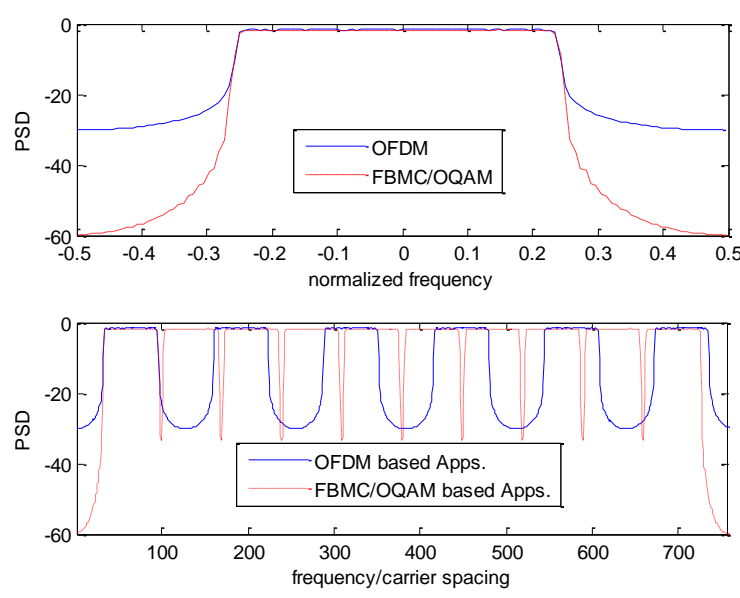

Fig. 2: Spectral side lobes and guard band

2) Channel equalization: The FBMC equalizer has to be adapted to cope with the intrinsic interference caused by multi-path channels. An analytical interference investigation has been reported in [8]. A linear multi-tap equalizer is proposed based on the frequency sampling technique [9]. An enhanced linear multi-tap equalizer based on the minimum mean square error (MMSE) criterion takes the inter-carrier interference (ICI) from adjacent subcarriers additionally into account and achieves a significantly improved performance [10]. An equalizer based on decision feedback (DFE) is proposed in [11], and a novel low-complex equalizer is presented in [12].

3) Multi-antenna (MIMO) transmission: An innovative mitigating technique for Alamouti coding scheme and maximum likelihood detection (MLD) is presented in [13]. [14] introduced a scheme based on spatial diversity to cancel the inter-symbol interference (ISI) and ICI. For MIMO precoding schemes, signal-to-leakage and noise ratio (SLNR) based algorithm is proposed in [15], and a zero forcing (ZF) based precoding is investigated in [16].

\section{FBMC related projects}

In 2009, the first FBMC dedicated project named PHYDYAS [17] was conducted under EU FP7. Currently, FBMC is under investigation by the EU FP7 project METIS [18] which studies the technology enablers for fifth-generation mobile wireless system. In the mean time, the project EU FP7 EMPhAtiC [19] investigates FBMC based advanced techniques for multicarrier ad-hoc and cell-based communications. The EU FP7 project 5GNOW [20] also considers FBMC as one of the candidate waveforms for $5 \mathrm{G}$.

\section{FBMC FOR 5G MOBILE SYSTEM}

5G mobile wireless system demands for a more flexible and scalable physical layer design to cope with the new use cases, device types and scenarios. The European METIS project elaborated on a set of scenarios foreseen for the information society beyond 2020 , which the $5 \mathrm{G}$ mobile wireless system should be able to address by providing novel and efficient solutions [21]. In the following section, two of these METIS 


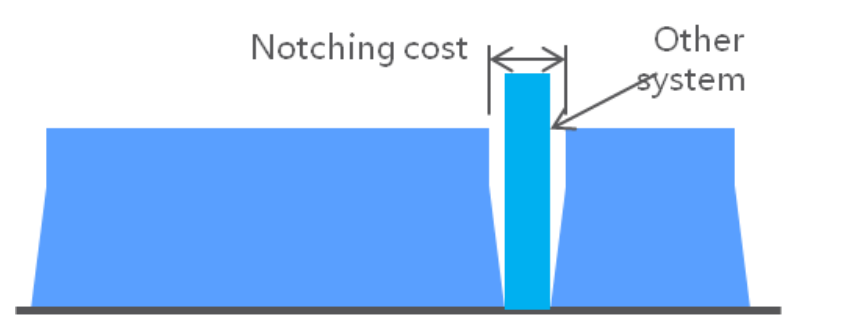

Fig. 3: Coexistence by notching FBMC signal

scenarios will be analyzed, and the potential benefits of using FBMC based modulation schemes will be explained.

\section{A. Great service in a crowd}

In this scenario, large crowds of people access the wireless network simultaneously, requesting different types of services that may obey different standards, potentially even from different operators. At the same time, there may be a multitude of machine type devices accessing the network additionally, acting as agents for a large variety of new services, which may, for example, support facility operations, public safety features, tracking of goods, monitoring current conditions in local areas, etc. These machine type devices may themselves have highly diverse requirements as well, encompassing low-cost batterypowered sensors as well as power-plugged complex controllers. Moreover, the particular demand in traffic for the different services mentioned may vary significantly over the day, not only depending on varying user densities. Therefore, the traffic situation - and thus the service distribution - is expected to be dynamically changing. In this scenario, we thus conclude to encounter at anytime a colorful mix of services with substantially different requirements, ranging from lowcost narrow-band to broad-band multimedia. 5G mobile wireless system must guarantee the coexistence of all these services while ensuring an efficient use of the scarcely available spectrum resources.

To solve the spectrum bottleneck, advanced spectrum sharing techniques can be adopted in this scenario, such as Cognitive Radio (CR) concept. In order to avoid severe interference between different radio services, there is usually a spectrum mask to be fulfilled. Since FBMC using appropriate prototype filters exhibits very low out-of-band leakage (as shown in Fig. 2 top), it can easily adapt to any kind of spectrum mask by simply switching off a set of adjacent subcarriers, which is also named "spectrum notching" (Fig. 3). Thanks to the steep power roll-off in frequency, the spectral leakage into adjacent frequency bands is minimized, and spectral guard bands can be reduced to a minimum. Hence, with the improved Power Spectral Density (PSD) shape, FBMC/OQAM based applications, as depicted in Fig. 2 bottom, enable the most efficient usage of fragmented spectrum resources while still respecting the required adjacent channel leakage ratio (ACLR). Moreover, link level spectral efficiency is further enhanced compared to OFDM, as no CP is needed.

\section{B. Best experience follows you}

This scenario aims at providing mobile radio services with high quality of experience to the users wherever they are, including
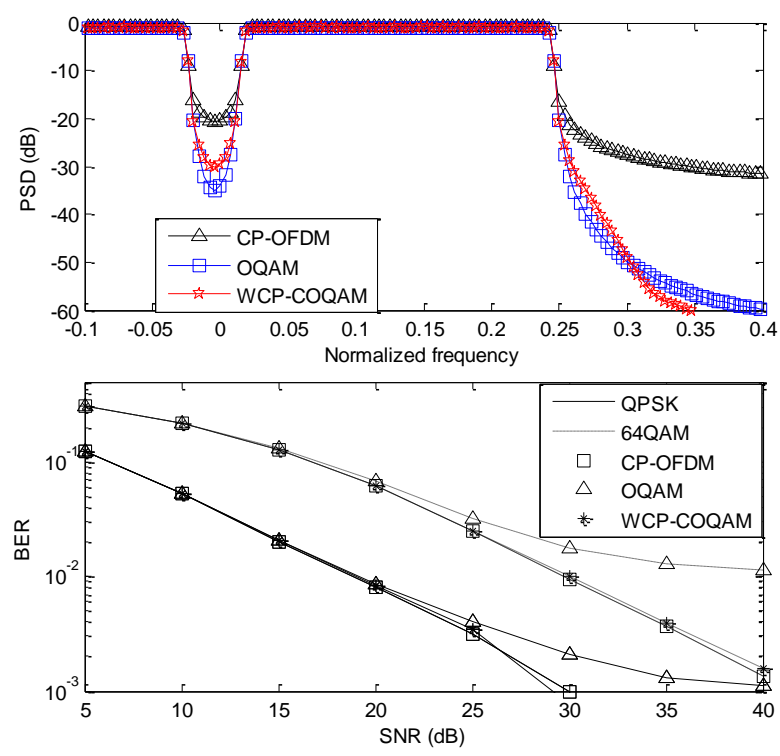

Fig. 4: PSD and BER evaluation for WCP-COQAM (from [22])

(and emphasizing) high mobility environments. Due to the Sinc-shaped spectrum in OFDM, Doppler shift and carrier frequency offset (CFO) effects can lead to severe interference between adjacent subcarrier signals.

By using FBMC with well localized pulse shapes, the effects of ICI can be strongly reduced and also confined in their range. Should ICI compensation still be necessary, compensation algorithms can then be designed to be more efficient, since only subcarriers in the direct neighborhood need to be considered. Furthermore, it is possible to optimize the pulse shape to a specific doubly-dispersive channel if the channel statistics (i.e. channel scattering function) are known to the system (see also section IV.C). At the same time, by relying on the high robustness of the FBMC waveform with respect to time and frequency jitter, the overall reliability of the system can also be improved for critical communication. Relevant test cases can be found in the field of real-time remote computing for mobile terminals, focusing on traffic efficiency and safety with assistive automotive technologies.

\section{CHALlENGES AND PROPOSED SOLUTIONS}

Although lots of progress has been achieved in FBMC prior art, still some challenges remain that deserve further investigations. In the following, we name three challenges, which have always been considered as bottlenecks for FBMC systems in the literature. In this section, we point out the most recent research progress towards potential solutions.

\section{A. Challenge \#1: Orthogonality Issue}

As elaborated briefly in Section II, FBMC/OQAM transmission schemes inherit an intrinsic interference in timedispersive channels due to their waveforms overlapping in time domain and the absence of a CP. In order to mitigate these QoS-degrading effects, suitable equalization and interference cancellation (IC) schemes have to be employed. As Alamouti and spatial multiplexing techniques are currently used in commercial OFDM-based systems such as LTE, the 


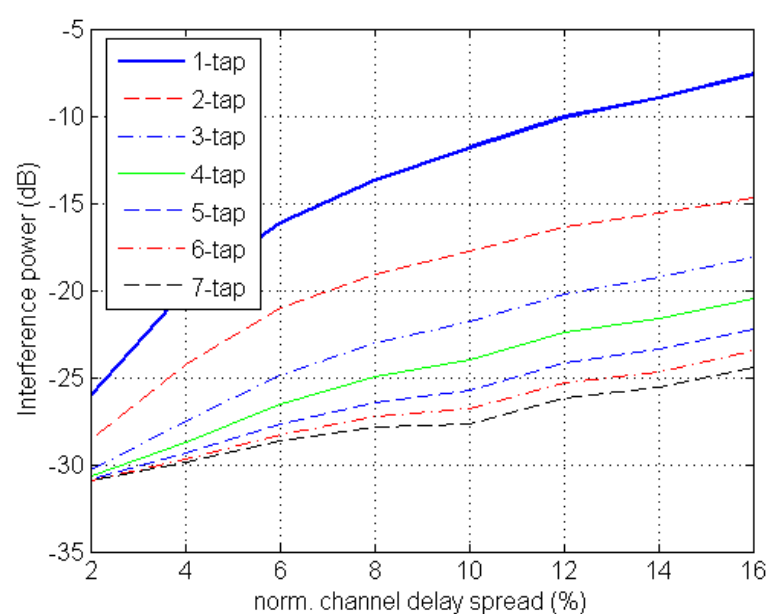

Fig. 5: Achievable SIR performance for multi-tap equalizer

investigation of MIMO in the proposed system is essential, especially with high level modulation alphabets. The objective is to provide efficient and relatively simple equalization and IC algorithms, which are suitable for both single antenna (SISO) and MIMO applications in time-dispersive channels.

The recent research outcome addressing these aspects contains different solutions. One solution, the so-called Windowed CP-based Circular OQAM (WCP-COQAM) [22], turns the traditional FBMC/OQAM continuous processing to a block processing by replacing linear sub-carrier filtering with circular sub-carrier filtering. With this modification, a CP can be easily inserted to each block, consequently relieving the equalizer effort. In addition, a particular windowing process is applied to effectively ensure three requirements: 1 ) the signal's PSD after the CP insertion can still remain at a similar level as that of FBMC/OQAM; 2) the windowing does not ruin orthogonality; 3) the windowing does not affect the spectral efficiency. Based on the reported results shown in Fig. 4, it is stated that the WCP-COQAM can completely solve the orthogonality problem [22].

Addressing equalization for continuous transmission of FBMC symbols following the conventional way (i.e. without any block structure), the multi-tap equalizer has been proposed as a suitable solution to combat the interference caused by long delay spread channels. Its complexity depends linearly on the number of filter taps used per subcarrier signal. Thus, from practical perspective, the number of filter taps should be kept to a minimum. However, information on the number of taps required to achieve a reasonable system performance in practice cannot yet be found in the literature. Hence, investigations have been carried out to evaluate the linear MMSE equalizer in [10] with respect to its achievable performance measured by the signal to interference ratio (SIR) in severe delay spread channels. Since the equalizer chosen takes into account both ISI from successive symbols as well as ICI from the adjacent subcarriers, it is considered the one achieving best performance from all linear solutions.

For the evaluation, a Rayleigh fading channel environment has been assumed with a uniform power delay profile (PDP).

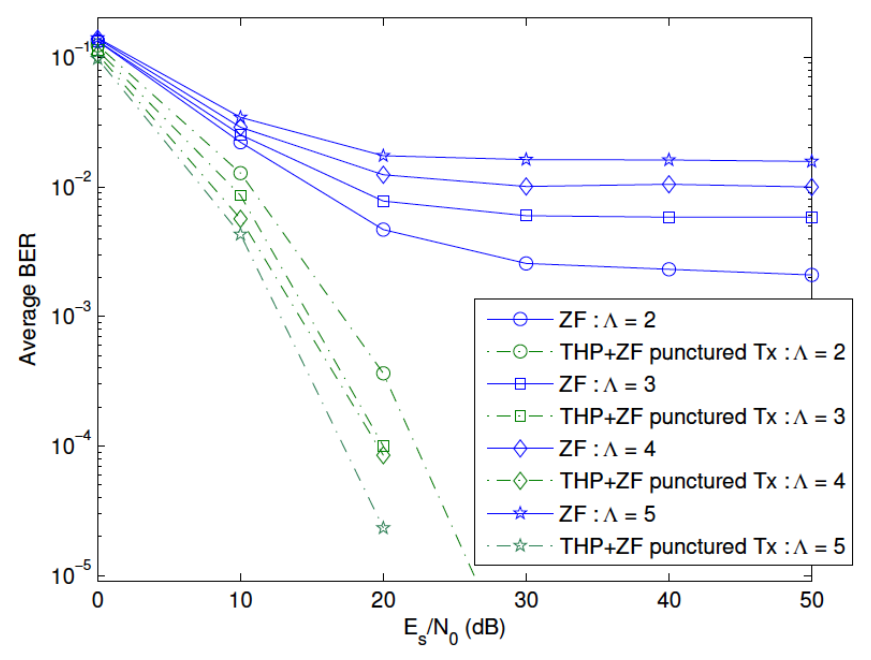

Fig. 6: Avg. BER performance with $M=16, L=63, r_{\max }=3, \varepsilon=1$.

This channel represents worst case conditions which are usually not experienced in practice; however, it allows for useful assessments of the equalizer's capability. The PHYDYAS pulse shape [7] has been applied with overlapping factor $K=4$. Since OQAM signaling has been used, the filter operates on a sampling period of $\mathrm{T} / 2$, with $\mathrm{T}$ being the symbol period equal to the inverse subcarrier spacing. Evaluation results are shown in Fig. 5, where the interference power measured on average per subcarrier relative to the average signal power has been plotted versus the maximum delay spread of the channel, which is given relative to $T$. The uppermost curve represents the performance achievable with a single-tap equalizer, which corresponds to the standard solution used in OFDM context. It can be seen that the SIR falls below $20 \mathrm{~dB}$ already at a relative delay spread of $4 \%$. Applying the multi-tap equalizer can improve the SIR performance substantially, where the major share of this gain is already attained by using two taps only. By noting that long delay spread channels occur rather in long-distance scenarios, where the operating range of the signal to noise ratio (SNR) is at 15 to $20 \mathrm{~dB}$ at maximum, the major conclusion to be drawn from this evaluation is that a 2-tap equalizer provides sufficient performance in most cases. In extreme cases, a 3-tap equalizer may be applied, which can provide an additional SIR gain of roughly $4 \mathrm{~dB}$.

As identified earlier, the application to MIMO with promising performance is crucial, if FBMC is to be considered a suitable contender to OFDM as used in LTE type systems. Motivated by this, the bit error rate (BER) performance of a MIMO FBMC system utilizing $M$ subcarriers with transmitter and receiver each equipped with $\mathrm{N}_{\mathrm{A}}$ antennas is analyzed comprehensively, and it is shown that the system can achieve low error rates [23]. For the receiver processing techniques discussed, we assume the receiver has perfect channel state information (CSI). For the precoding methods discussed, we assume perfect CSI is available at both the transmitter and the receiver. This assumption is to be relaxed later on. The complexity and performance trade off is considered for these cases. In particular, punctured Tomlinson Harashima 


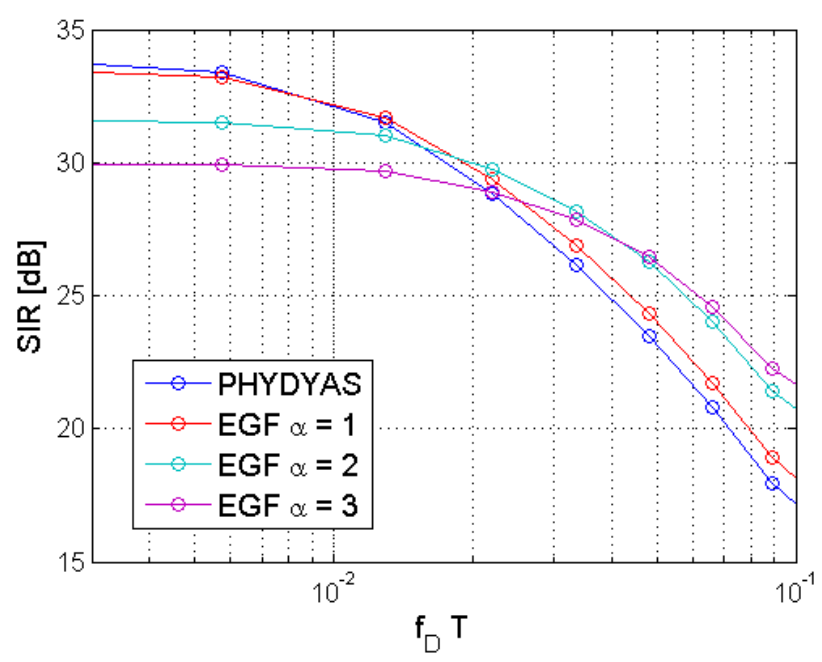

Fig. 7: SIR of different pulse shaped in 2D channel

precoding (THP) technique is proposed which shows feasibility of FBMC with significantly lower BER. The approach is to remove the error floor by precoding, at a slight loss of throughput, by not using one or more antennas on some subcarriers. We call this puncturing. We assume that all antenna paths undergo independent fading. A time-invariant Rayleigh fading channel is considered, where the channel impulse response spans $\Lambda$ sampling intervals. The sampling corresponds to original symbol duration before FBMC mapping. The filter length is $\mathrm{L}$. We can limit the maximum interference on a symbol to $\varepsilon$ with $r$ iterations in precoding. Signal amplitude without interference is set to unity. Fig. 6 shows the average BER, parameterized by the number of channel taps $\Lambda$ with first tap variance is unity and the rest is of equal strength, giving an overall variance of 1.2. The throughput loss is around 3\%. A comparison to $\mathrm{ZF}$ equalizer is also shown. Results show that with the novel proposal for MIMO transmission in FBMC system, the error floor can be effectively removed.

\section{B. Challenge \#2: Packet transmission}

In theory, FBMC/OQAM is able to achieve full time/frequency efficiency through the use of OQAM. However, this holds only in case of symbol sequences with infinite length. In realistic scenarios, data transmission is divided into smaller time direction chunks (e.g. LTE: a single TTI spans $1 \mathrm{~ms}$ ). Then, ramp-up and ramp-down times at the edges of these intervals caused by filtering are reducing the actual efficiency. This depends on the number of symbols and on the average number of subcarriers of a typical user allocation (with a smaller average allocation and given usable subcarriers, more users are allocated to the same transmission time interval (TTI)). Hence, this may become a major issue in applications such as machine-type communications, where the packets to be transmitted are expected to be rather short. Some solutions have been proposed to overcome this problem, such as burst truncation [24]. It has been investigated that spectral efficiency loss can be recovered with justifiable performance degradation. Another alternative solution, which was reported in [25], aims to use a package transmission concept with special processing at the edge side. By this way, no truncation is needed, which avoids the performance loss. Nevertheless, since this method is only valid for short filter lengths, further investigation is needed to overcome this filter length limit. Recently, the circularly convolved FBMC concept has been presented which completely breaks through this bottleneck. In this respect, two solutions were independently investigated, but intend to go towards similar direction. The first solution is the before-mentioned WCP-COQAM (see section IV.A), the second has been introduced under the name "weighted circularly convolved FBMC" [26]. The block structure can be chosen to cover any number of symbol periods $\mathrm{T}$ larger or equal to one, so it can be adapted for any short packet length, while requiring a small overhead for the windowing only. Since both methods are based on a block-processing based modulation, the packet transmission issue can be naturally solved.

\section{Challenge \#3: Waveform and transceiver adaptation}

Compared to conventional OFDM systems, where the system configuration is fixed to a "one-fits-all" solution fulfilling the requirements of the entire set of targeted services and applications as a best compromise, the FBMC signal structure offers a high degree of flexibility to the system design: If pulse shapes with steep power roll-off in frequency domain are used, the transmission band can be easily partitioned into independent sub-bands, which may be individually configured according to the particular demands of a service to be provided. Selecting the most suitable pulse shape would be one configuration option. This flexibility of the FBMC structure thus enables full adaptivity of waveform and transceiver design responding to any kind of requirements given by a service or application. A system design and performance evaluation for such a flexible FBMC system is currently under study.

Concerning pulse shape selection, it is well known that an adaptation to the channel's energy spread in time and frequency will improve the transmission performance, since ISI and ICI can be minimized. However, information on quantitative potential performance gains in practical scenarios is not yet found in the literature. Recent investigations addressing this question [27] are briefly presented here: For evaluations, the IOTA pulse based on extended Gaussian functions (EGF) [28] has been selected, as it allows adapting the pulse shape to the $2 \mathrm{D}$ channel scattering function by simply adjusting a parameter $\alpha$. This parameter affects the power distribution of the pulse in the time/frequency space as follows:

$\alpha=2$ the power is isotropically spread in the time/frequency grid for OFDM/OQAM, which is spanned by subcarrier spacing $F_{0}$ and sampling period T/2.

$\alpha>2$ the pulse is broadened in frequency, making it less prone to frequency spreads / shifts, as imposed by Doppler effects

$\alpha<2$ the pulse is broadened in time domain, making it less prone to time spreads / shifts, as imposed by delay spread channels 
Based on the ambiguity function, which covers the pulse shape and its distortion due to the delay and Doppler spread channel [29], the pulse shape's reconstruction quality has been evaluated in terms of an SIR measure. Results are shown in Fig. 7 for a channel with exponentially decaying PDP at a constant root mean square (RMS) delay spread of $0.01 \mathrm{~T}$ and a variable Doppler spread with Jakes' spectrum and normalized Doppler frequency $f_{\mathrm{D}} \mathrm{T}$. We can observe that at low Doppler, IOTA with $\alpha=1$ and PHYDYAS pulse achieve the best performance, exceeding that of IOTA with $\alpha=3$ by $3 \mathrm{~dB}$. For high Doppler, however, this latter pulse excels the performance of PHYDYAS by roughly $5 \mathrm{~dB}$. In line with the theory, when following the transition between these two extremes, we observe that there is an area where IOTA with $\alpha=2$ provides the best SIR performance. However, compared to the other two IOTA candidates, this pulse can provide an additional SIR gain of merely half a $\mathrm{dB}$. These results indicate that considerable SIR gains can be achieved by adapting the pulse shape to the channel, in particular if severe Doppler distortions are present. The findings justify considering pulse shape adaptation also in practical systems.

\section{CONCLUSION}

FBMC is considered a potential candidate for the $5 \mathrm{G}$ air interface, as its inherent properties provide the flexibility needed to respond to the diverse service requirements expected in future communication scenarios. After presenting a brief overview on the current state of the art in FBMC research, the potential of this technology has been illustrated in the light of selected scenarios for future communication developed by the FP7 METIS project. Three main challenges have been highlighted where research efforts are still needed to render FBMC an eligible candidate for the $5 \mathrm{G}$ air interface, which are summarized as: 1) the orthogonality issue brought up in time-dispersive channels, 2) the overhead problem in packet transmission and 3) the realization of waveform and transceiver adaptation in a practical system. For each of these challenges, approaches and solutions covering latest results of the METIS radio link research have been presented.

\section{ACKNOWLEDGEMENT}

Part of this work has been performed in the framework of the FP7 project ICT-317669 METIS, which is partly funded by the European Union. The authors would like to acknowledge the contributions of their colleagues in METIS, although the views expressed are those of the authors and do not necessarily represent the project.

\section{REFERENCES}

[1] P. Siohan, C. Siclet and N. Lacaille, "Analysis and design of OFDM/OQAM systems based on fillterbank theory", IEEE Trans. on Signal Processing, vol. 50, pp. 1170-1183, May 2002.

[2] H. G. Feichtinger and T. Strohmer, Gabor analysis and algorithms, Birkhäuser, 1998.

[3] B. Le Floch, M. Alard and C. Berrou, "Coded orthogonal frequency division multiplex", Proceedings of the IEEE, vol. 83, no. 6, pp. 982996, Jun. 1995
[4] M.G. Bellanger. "Specification and design of a prototype filter for filterbank based multicarrier transmission". In Acoustics, Speech, and Signal Processing, 2001.

[5] M. Rossi, J.Y. Zhang and W. Steenaart, "Iterative constrained least squares design of near perfect reconstruction pseudo QMF banks", CCECE'96, May, 1996.

[6] D. Pinchon, P. Siohan and C. Siclet, "Design techniques for orthogonal modulated filterbanks based on a compact representation', IEEE Trans. on signal processing, 52(6):1682-1692, Jun. 2004.

[7] FBMC Physical layer: a primer, PHYDYAS EU FP7 Project, 2010.

[8] H. Lin and P. Siohan, "Capacity analysis for PLC with different multicarrier modulations", IEEE Trans. on Power Delivery, vol 25, no. 1, pp. 113-124, Jan. 2010.

[9] T. Ihalainen, T.H. Stitz, M. Rinne and M. Renfors, "Channel equalization in filter bank based multicarrier modulation for wireless communications", EURASIP, 2007 (ID 49389), Aug. 2007.

[10] A. Ikhlef and J. Louveaux, "An enhanced MMSE per subchannel equalizer for highly frequency selective channels", SPAWC'09, 2009

[11] H. Lin, P. Siohan, P. Tanguy and J.P. Javaudin, "An analysis of EIC for OFDM/OQAM', Journal of Communications, vol. 4, no. 1, pp. 52-60, Feb. 2008 .

[12] G. Ndo, H. Lin and P. Siohan, "FBMC/OQAM equalization: exploiting the imaginary interference', PIMRC'12, Sept.0 2012.

[13] R. Zakaria,D. Le Ruyet, "A Novel Filter-Bank Multicarrier Scheme to Mitigate the Intrinsic Interference: Application to MIMO Systems,' Wireless Communications, IEEE Transactions on , vol. 11, no.3, pp. 1112- 1123, Mar. 2012.

[14] Nizar Zorba, Faouzi Bader, "Spatial Diversity Scheme to Efficiently Cancel ISI and ICI in OFDM-OQAM Systems," Journal of Computer Systems, Networks, and Communications, vol. 2010, 2010

[15] Wang Jingjing, Xianzhong Xie, Qian Zhang, "A Way to Reduce ICI of Multi-user MIMO-OFDM System with Precoding," International Conference on Advanced Computer Control, 2009. ICACC '09., pp. 134- 137, 22-24 Jan. 2009.

[16] D. Ben Cheikh, J.-M. Kelif, M. Coupechoux, P. Godlewski, "Multicellular Zero Forcing Precoding Performance in Rayleigh and Shadow Fading," Vehicular Technology Conference (VTC Spring), 2011 IEEE 73rd, pp. 1-5, 15-18 May 2011.

[17] FP7 PHYDYAS Project. http://www.ict-phydyas.org

[18] FP7 METIS 2020 Project https://www.metis2020.com

[19] FP7 EMPhAtiC Project, http://www.ict-emphatic.eu/

[20] FP7 5GNOW Project, http://www.5gnow.eu/

[21] METIS deliverable D1.1, "Scenarios, requirements and KPIs for 5G mobile and wireless system".

[22] H. Lin and P. Siohan, "An advanced Multi-Carrier Modulation for Future Radio Systems”, ICASSP, May 2014.

[23] Soysa, M, Rajatheva, N., Latva-aho, M., "Linear and Non-linear Transceiver Processing for MIMO-FBMC Systems”, ICC, Jun. 2014.

[24] PHYDYAS deliverable D2.1, 'Data-aided synchronization and initialization (single antenna)', chapter 6.1

[25] Y. Dandach and P. Siohan, "Packet Transmission for Overlapped Offset QAM', IEEE Int. Conf. on Wireless Communications and Signal Processing, Suzhou (China), Oct. 2010.

[26] M. J. Abdoli, M. Jia, J. Ma, "Weighted circularly convolved filtering in OFDM/OQAM”, IEEE PIMRC, pp. 657-661, 2013

[27] M. Fuhrwerk, J. Peissig, M. Schellmann, "Channel adaptive pulse shaping for OQAM-OFDM systems", submitted to EUSIPCO 2014

[28] P. Siohan and C. Roche, "Cosine-modulated filterbanks based on extended Gaussian functions", IEEE Trans. on Signal Processing, vol. 48, pp. 3052-3061, Nov. 2000

[29] J. Du, S. Signell, "Novel preamble-based channel estimation for OFDM/OQAM system”, IEEE ICC 2009 